\title{
TROMPING GAMES: TILING WITH TROMINOES
}

\author{
SAÚL A. BLANCO AND AVIEZRI S. FRAENKEL
}

\begin{abstract}
The game of Domineering is a combinatorial game that has been solved for several boards, including the standard $8 \times 8$ board. We create new partizan - and some impartial - combinatorial games by using trominos instead of and along with dominoes. We analyze these Tromping games for some small boards providing a dictionary of values. Further, we prove properties that permit expressing some connected boards as sums of smaller subboards. We also show who can win in Tromping for some boards of the form $m \times n$, for $m=2,3,4,5$ and infinitely many $n$.
\end{abstract}

\section{INTRODUCTION}

1.1. Outline. The game of Domineering was invented by Göran Andersson around 1973, according to $[\mathrm{BUvdH}],[\mathrm{Con}]$, and [Gar]. The two players in Domineering alternately tile a board using a regular domino (a $2 \times 1$ tile). The players are usually called Vertical (or Left) and Horizontal (or Right). They place their tiles, without overlapping, vertically and horizontally, respectively. The player making the last move wins. The game is partizan, since the set of moves is different for each player. Conway [Con]; and Berlekamp, Conway, and Guy [BCG] have computed the value of Domineering for several small boards, not necessarily rectangular. D.M. Breuker, J.W.H.M Uiterwijk and H.J. van den Herik [BUvdH] have determined who wins the game of Domineering for additional boards. In particular, they showed that the first player can win on the classical $8 \times 8$ board, which was the original game presented by Andersson.

In this paper we investigate the game of Tromping, which is played as Domineering, but with larger tiles instead of, or along with, dominoes. The additional polyominoes are trominoes, also called triominoes. There are 2 trominoes: a "straight" tromino, i.e., a $3 \times 1$ tile, dubbed I-tromino; and an L-shaped tromino, i.e., a $2 \times 2$ tile with one square removed, dubbed L-tromino.

Any nonempty subset of a rectangular board is called a board or subboard in this paper. The boundaries of its squares are vertical and horizontal only. In Tromping, Vertical and Horizontal place dominoes and I-trominoes vertically and horizontally respectively; the L-tromino can be placed by either player in either one of its four orientations (an "impartial" tromino).

We assume that the reader is familiar with the basic theory of combinatorial games, which can be found in $[\mathrm{BCG}],[\mathrm{Con}]$ and $[\mathrm{ANW}]$. A concise 18-page summary with the basic results in combinatorial game theory can be found in [Con77]. We recall that for a partizan game $G$, Vertical (Left) can win if $G>0$, Horizontal (Right) can win if $G<0$, the second player can win if $G=0$, and the first player can win if $G \| 0$ ( $G$ is fuzzy (incomparable) with 0$)$. In particular, Horizontal wins as second player if $G \leq 0$, and as first player if $G \triangleleft 10$.

In Section 2 we create new partizan combinatorial games, variations of Domineering, by admitting the use of larger tiles. For each game we provide dictionaries of values - where squares that cannot be used during the play (not part of the board) are painted black. We also prove some properties that allow one to express the value of a "connected" board $B$ as the sum of the values of subboards $B_{1}$ and $B_{2}$.

2000 Mathematics Subject Classification. Primary 91A46.

The first-named author was supported, in part, by NSF grant DMS-0555268. He thanks A. S. Fraenkel for introducing him to the world of Combinatorial Games, and D. J. Crandall for valuable computer support. 
Normally only disjoint subboards form a sum. We point out that all our games are acyclic and short; that is, there are no repetitions and only finitely many positions .

In Section 3 we discuss some ideas from number theory that can be used to determine the winner of our variations for particular families of boards. These ideas seem to be implicit in Lachmann, Moore, and Rapaport [LMR].

In Section 4 we provide several results on our variations. For example, we apply the results from number theory discussed in Section 3 to determine the winner for particular boards of the form $m \times n$ for $m=2,3,4,5$ and infinitely many $n$. We also include other results about a new impartial game, M-Tromping.

Finally, for convenience, whenever no confusion arises, we do not distinguish between the board on which the players are tiling and the actual game.

1.2. Computation of the values. Values and outcomes of the games were computed by constructing plug-ins for Siegel's Combinatorial Game Suite (CGS) [Sie]. Such plug-ins were needed in order for CGS to understand the rules of the variations of Domineering. No changes were done to the code of CGS. In the computation of the values of the games, CGS considers all possible positions that can be reached in a game without considering efficiency. This approach differs from that of $[\mathrm{BUvdH}]$ where the authors cut off some positions using an $\alpha-\beta$ search technique to determine the winner.

For the computer experiments, we used a Dell PowerEdge 1750 server with dual $3 \mathrm{GHz}$ Xeon processors and $4 \mathrm{~GB}$ of memory running Linux 2.4.21 and a Sun Enterprise 420R server with quad UltraSPARC processors and $4 \mathrm{~GB}$ of memory running Solaris 8 .

For those values too long to write down, we only present the winner, according to whether $G$ is positive, negative, zero, or fuzzy with 0 . For example, the value corresponding to the game of I-Tromping (see below) played on the $6 \times 6$ board is

$$
\pm(\{2|1| \mid 1 / 2\},\{\{6|5||9 / 2||| 2|1| \mid 1 / 2\},\{6|5||9 / 2||| 4 \mid 0\} \mid\{4|0|||-1 / 2||-1 \mid-2\},\{7 / 2|| 3|2|||-1 / 2||-1 \mid-2\}\})
$$

which is fuzzy with 0 , so the first player wins.

Throughout the paper, we use the notations ${ }^{*}=\{0,0\}$, and $n^{*}=n+^{*}=\{n \mid n\}$ for all integers $n \geq 1$.

\section{New COMbinatorial Games}

2.1. I-Tromping. In our first game, we substitute the domino by an I-tromino. Its rules are exactly the same as for Domineering: the two players, Vertical and Horizontal, tile alternately vertically and horizontally, respectively. Overlapping is not permitted. The player making the last move wins. It might appear that I-Tromping is simply a scaled version of Domineering, but this is not the case for boards that are not rectangular. For square boards there is some resemblance, though: In Domineering, the $2 \times 2$ and $3 \times 3$ boards both have value \pm 1 ; for I-Tromping the $3 \times 3$ and $5 \times 5$ boards both have value \pm 2 .

TABLE 1. Values of I-Tromping for small rectangular boards.

\begin{tabular}{|c|c|c|c|c|c|c|}
\hline & 1 & 2 & 3 & 4 & 5 & 6 \\
\hline 1 & 0 & 0 & -1 & -1 & -1 & -2 \\
2 & 0 & 0 & -2 & -2 & -2 & -4 \\
3 & 1 & 2 & \pm 2 & $\{3 \mid-3 / 2\}$ & $\left\{4 \mid-1,-1^{*}\right\}$ & $\{4|0|||-1 / 2||-1 \mid-2\}$ \\
4 & 1 & 2 & $\{3 / 2 \mid-3\}$ & $\pm 5 / 2$ & $\left\{3 \mid-2,-2^{*}\right\}$ & $\{3|-3 / 2|||-7 / 4||-3 \mid-4\}$ \\
5 & 1 & 2 & $\left\{1,1^{*} \mid-4\right\}$ & $\left\{2,2^{*}-3\right\}$ & \pm 2 & $\left\{-3||-3,-3^{*} \mid-8\right\}$ \\
6 & 2 & 4 & $\{2|1||1 / 2||| 0 \mid-4\}$ & $\{4|3||7 / 4||| 3 / 2 \mid-3\}$ & $\left\{8\left|3,3^{*}\right| \mid 3\right\}$ & $\mathrm{F}$ \\
\hline
\end{tabular}




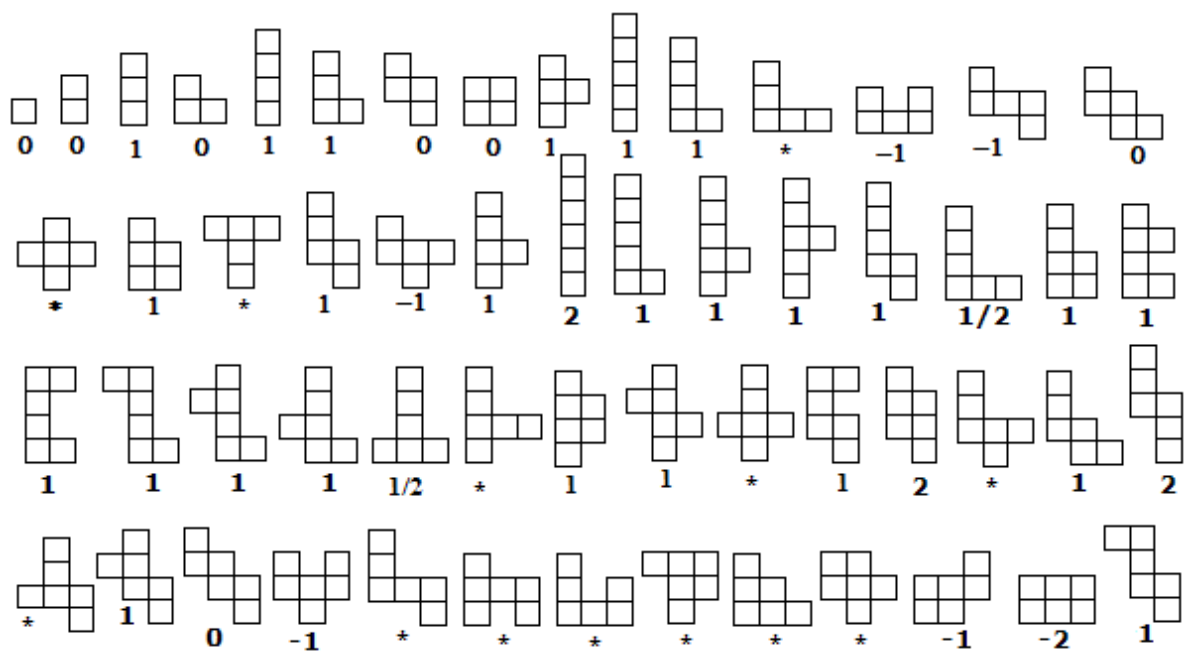

FIGURE 1. I-Tromping values for boards up to 6 squares.

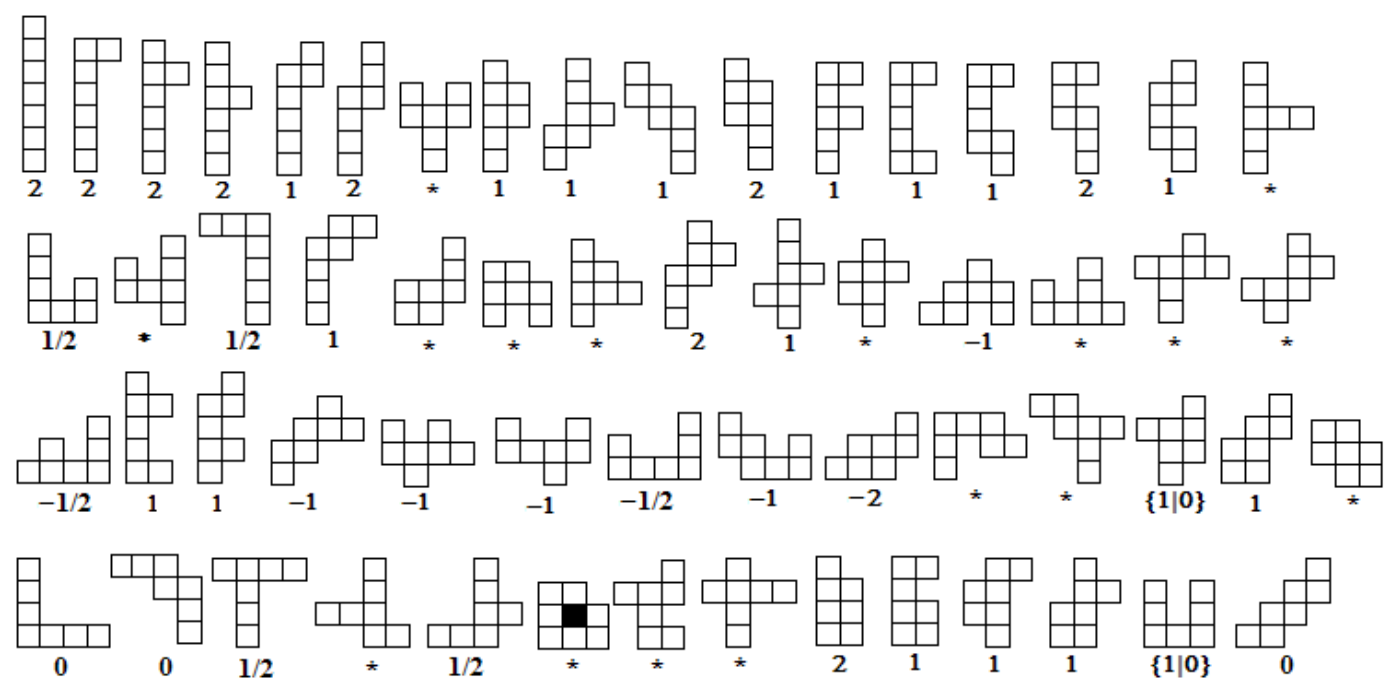

Figure 2. I-Tromping values for some boards with 7 squares.

Figure 1 gives the values of I-Tromping for boards up to six squares, including the 35 boards with 6 squares, excluding their negatives obtained by a right angle turn. Figure 2 displays 59 boards with seven squares and their values. According to [BCG], Vol. 1, Ch. 5, Extras, there are 108 boards with seven squares.

We enumerate the rows of a board from top down and its columns from left to right, as is common when labeling the rows and columns of a matrix. Since a board need not be rectangular, a row or column can be formed by only one square.

Table 1 depicts the value of I-Tromping for some rectangular boards. We have omitted "messy" values - those that take considerable space to express. Instead, we have used F to indicate that the first player wins; V, that Vertical wins; and H, that Horizontal wins. This notation is also used in the tables (below) containing the values of D-Tromping and L-Tromping (explained below) for small rectangular boards. 
Definition 2.1. A board $F$ is said to be concatenated to a board $G$ if a domino can be placed horizontally so that its left square tiles a square of $F$ and its right square tiles a square of $G$. Notation: $F G$. If the connecting domino is placed in row $i$ of $F$ and row $j$ of $G$, we also use the notation $F G_{(i, j)}$.

Definition 2.2. Let $F G_{(i, j)}$ be a concatenation of the board $F$ to $G$. If each column of $F G_{(i, j)}$ intersects only one of the two boards, then $F$ is $(i, j)$-aligned to $G$. If $F$ is $(i, j)$-aligned to $G$ for all possible $(i, j)$, then we simply say that $F$ is aligned to $G$. Otherwise they are not aligned.

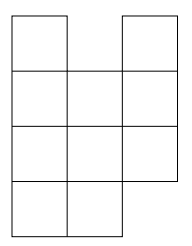

$A$

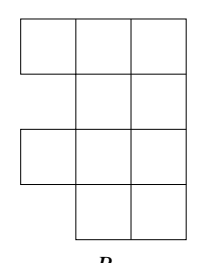

B
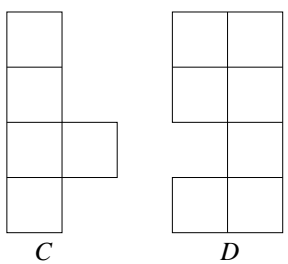

Figure 3. Aligned and non-aligned boards.

Figure 3 depicts an example where $A$ is $(1,1)$-aligned to $B$ but not $(4,1)$-aligned, as $A B_{(4,1)}$ will contain in its third column squares from both boards. Also, $C$ is not $(1,1)$-aligned to $D$, but it is $(3,1)$-aligned to $D$. On the other hand, $D$ is aligned to $C$.

Definition 2.3. A rectangular board is called 2-wide if it has a row with exactly two consecutive squares and no row or column with three consecutive squares.

Clearly any 2-wide board is a 0 -game in I-Tromping, since no player is able to move.

These definitions permit us to express some connected boards as sums of their subboards.

Proposition 2.4. Let $F$ be aligned to $G$, and $G$ aligned to $H$, where $G$ is 2-wide. Then in I-Tromping,

(a) $F G+G H \leq F G H$,

(b) If $F G=F$ then $F G H=F+G H$,

(c) If $G H=H$ then $F G H=H+F G$.

Proof. (a) It suffices to show that Horizontal can win as second player in $F G+G H-F G H$ (see Figure 4(a)). If Vertical begins by playing exclusively on $F$ or $H$, then Horizontal can respond by playing exclusively on $-F$ or $-H$ respectively, and conversely. Since $F$ is aligned to $G$, Vertical has no moves using squares from both of these boards, and the same holds for $G$ and $H$. The only way Vertical can use squares from two boards is on $-H$ and $-G$ or $-F$ and $-G$. Horizontal can counter these moves by using squares from $H$ and $G$ or $F$ and $G$, respectively. These are the only options of Vertical since $G$ is 2-wide, so no player can move exclusively on $G$ or move using squares from all three boards. Hence Horizontal can win.

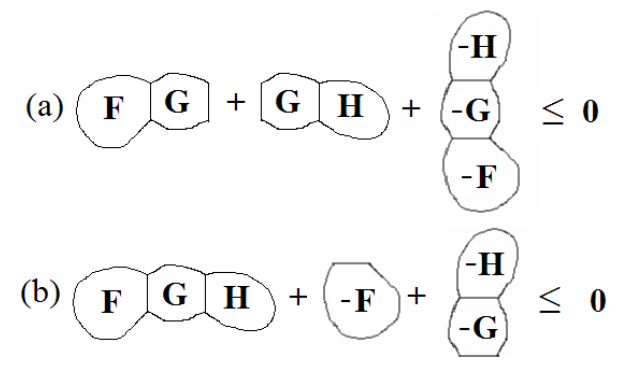

FiguRE 4. Illustrating the proof of Proposition 2.4. 
(b) We first prove that $F G H \leq F+G H$ by showing that $F G H-F-G H \leq 0$ (see Figure 4(b)). If Vertical, as first player, plays exclusively on $F$ or $H$, then Horizontal can respond by playing exclusively on $-F$ or $-H$, respectively, and conversely. Further, if Vertical plays using squares from $-H$ and $-G$, then Horizontal can move using squares from $H$ and $G$, respectively. Since these are the only options for Vertical, Horizontal can win.

To complete the proof, we use the result just proved and part (a):

$$
F G H \leq F+G H=F G+G H \leq F G H,
$$

and the result follows.

(c) The proof is the same as for (b).

Corollary 2.5. Let $F$ be aligned to $\square$, and $\square$ aligned to $H$. Then

(a) $F \square+\square H \leq F \square H$,

(b) If $F \square=F$ then $F \square H=F+\square H$,

(c) If $\square H=H$ then $F \square H=H+F \square$.

Proof. These are special cases of Proposition 2.4 (a) - (c) with $G=$

\section{Remarks.}

(1) The condition that the boards must be aligned is necessary: Figure $5($ a) depicts a case where $G$ is not aligned to $H$, with $G$ 2-wide. Proposition 2.4 (a) does not hold, for otherwise $*+\{0 \mid-1\}+1=$ $\left\{\left.1^{*}\right|^{*}\right\} \leq 0$, which is false since $\left\{\left.1^{*}\right|^{*}\right\}$ is positive.

(2) The condition that $G$ must be 2 -wide is also necessary. Figure 5 (b) exhibits a case where $G=0$ is not 2-wide and the proposition does not hold, since it is easy to verify that

$\{1 \mid-3\}+\{1 \mid 1 / 2\}=\{2|3 / 2||-2|-5 / 2\} \not \leq\{2|3 / 2||-2|-3\}$.

(a)

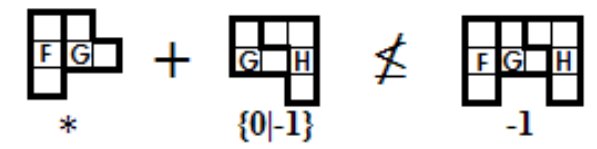

(b)

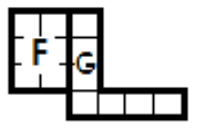

$\{\mathbf{1} \mid-\mathbf{3}\}$

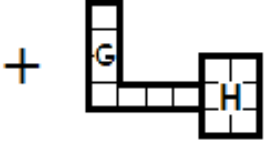

$\{\mathbf{1} \mid \mathbf{1} / \mathbf{2}\}$

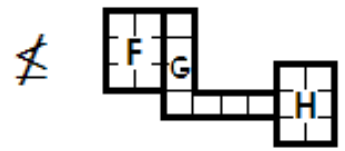

$\{2|3 / 2||-2|-3\}$

FiguRE 5. Boards alignment and 2-wideness are necessary.

(3) If $G \neq 0$, then Proposition 2.4 does not hold. Consider $F=G=H=$ the $3 \times 1$ tile. Then $F G H= \pm 2$ (Table 1), and $F G=G H=2$. Clearly, $2+2 \not \leq \pm 2$, so (a) does not hold.

(4) Proposition 2.4 holds also for playing with a "straight" $n$-polyomino (an $n \times 1$ tile), if we require $G$ to be $(n-1)$-wide. The proof of this claim is entirely analogous to the above. For increasing $n$, there is a growing set of $(n-1)$-wide boards $G$, for each of which Proposition 2.4 holds. For Domineering, however, Proposition 2.4 holds if and only if $G$ is the $1 \times 1$ tile (see also Proposition 2.6 below).

The last remark shows that for increasing size of the (smallest) tiling polyomino, the power of Proposition 2.4 increases, as it permits to express as sums a growing variety of boards that are not disjoint. This can already be observed for I-Tromping. In Figure 6 we have applied Proposition 2.4(b) to two 
different cases: The first one with $G$ being the $2 \times 2$ board, $F$ the $3 \times 3$ board, and $H$ the $3 \times 1$ tile. In the second case, we take $G$ to be a $2 \times 2$ board with one square removed. Note that $G$ need not be rectangular. From Figure 6 we see that

$$
\begin{aligned}
& \{2|1||-2|-3\}= \pm 2+\{0 \mid-1\} \\
& \pm(2)^{*}= \pm 2+*
\end{aligned}
$$

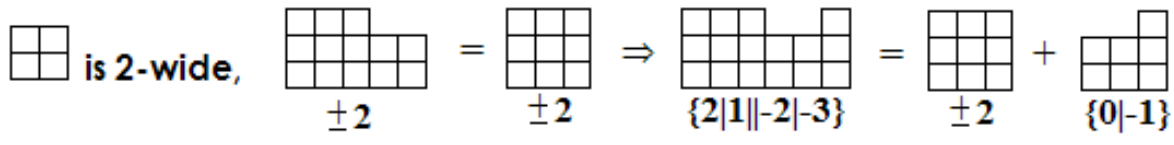

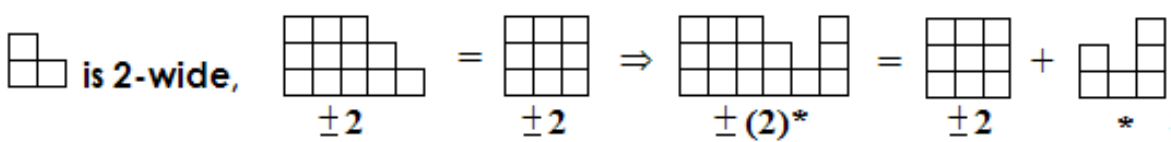

Figure 6. Two sample applications of Proposition 2.4 (b).

In general we cannot divide a "connected" board into pieces so that the value of the original board equals the sum of the values of the smaller boards, but here we can.

2.2. D-Tromping. Here Vertical and Horizontal alternate in tiling with either a domino or an I-tromino. The player making the last move wins. A dictionary of values for boards of up to six squares is depicted in Figure 7. Table 2 presents some of the values of rectangular boards.

Proposition 2.6. Let $F$ be aligned to $\square$, and $\square$ aligned to $H$. Then for D-Tromping we have,

(a) $F \square+\square H \leq F \square H$,

(b) If $F \square=F$ then $F \square H=F+\square H$,

(c) If $\square H=H$ then $F \square H=H+F \square$.

Proof. Same as Proposition 2.4 with $G$ replaced by $\square$.

This proposition holds also for Domineering; see [Con] Ch. 10.

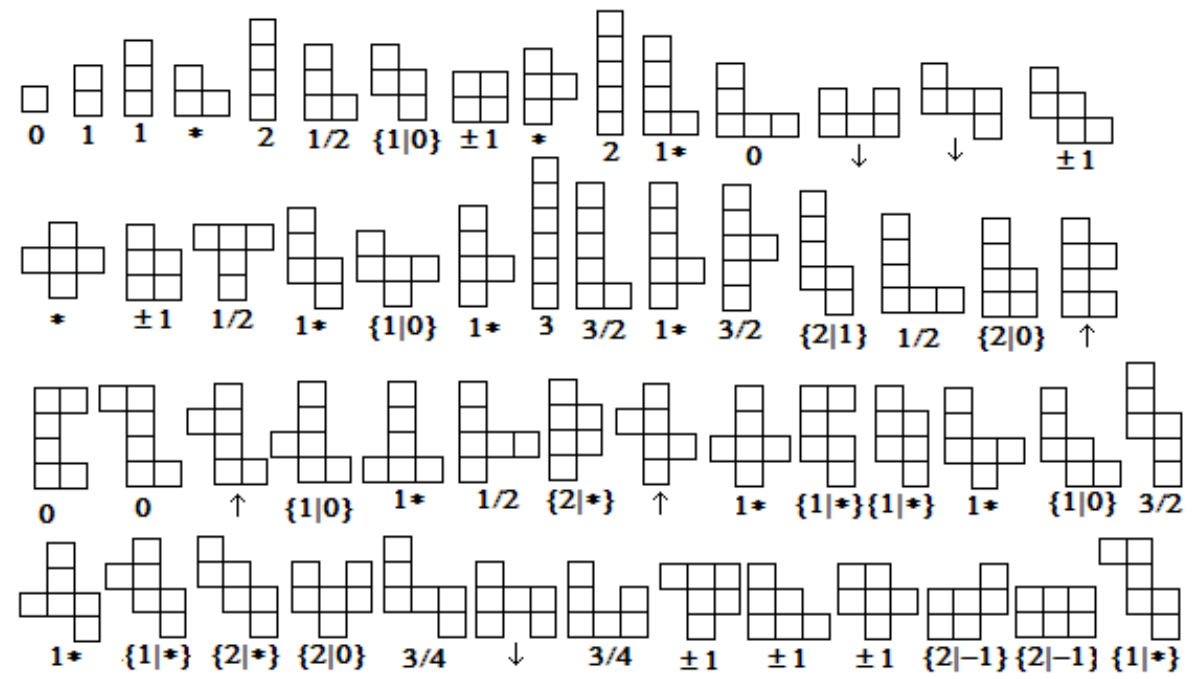

FiguRE 7. D-Tromping values for boards up to 6 squares. 
TABLE 2. Values of D-Tromping for small rectangular boards.

\begin{tabular}{|c|c|c|c|c|c|}
\hline & 1 & 2 & 3 & 4 & 5 \\
\hline 1 & 0 & -1 & -1 & -2 & -2 \\
2 & 1 & \pm 1 & $\{2 \mid-1\}$ & $\left\{2|0| \mid-1^{*}\right\}$ & $\{0,\{3 \mid 0\} \mid-1,\{0 \mid-2\}\}$ \\
3 & 1 & $\{1 \mid-2\}$ & \pm 2 & $\{2|-1| \mid-2 \uparrow\}$ & $\{-1,\{3 \mid-1\} \mid-7 / 4,\{-1 \mid-4\}\}$ \\
4 & 2 & $\left\{1^{*}|| 0 \mid-2\right\}$ & $\{2 \downarrow|| 1 \mid-2\}$ & $\pm 1^{*}$ & $\mathrm{~F}$ \\
5 & 2 & $\{1,\{2 \mid 0\} \mid 0,\{0 \mid-3\}\}$ & $\{7 / 4,\{4 \mid 1\} \mid 1,\{1 \mid-3\}\}$ & $\mathrm{F}$ & $\mathrm{F}$ \\
\hline
\end{tabular}

2.3. L-Tromping. In this game we tile with an L-tromino in addition to the I-tromino and domino. The L-tromino adds a total of 4 new moves to the set of moves of each player, since it can be rotated and placed in 4 different positions on a rectangular board. A dictionary of values of this game for small boards is exhibited in Figures 8 and 9, and Table 3 depicts the values of L-Tromping for some rectangular boards.

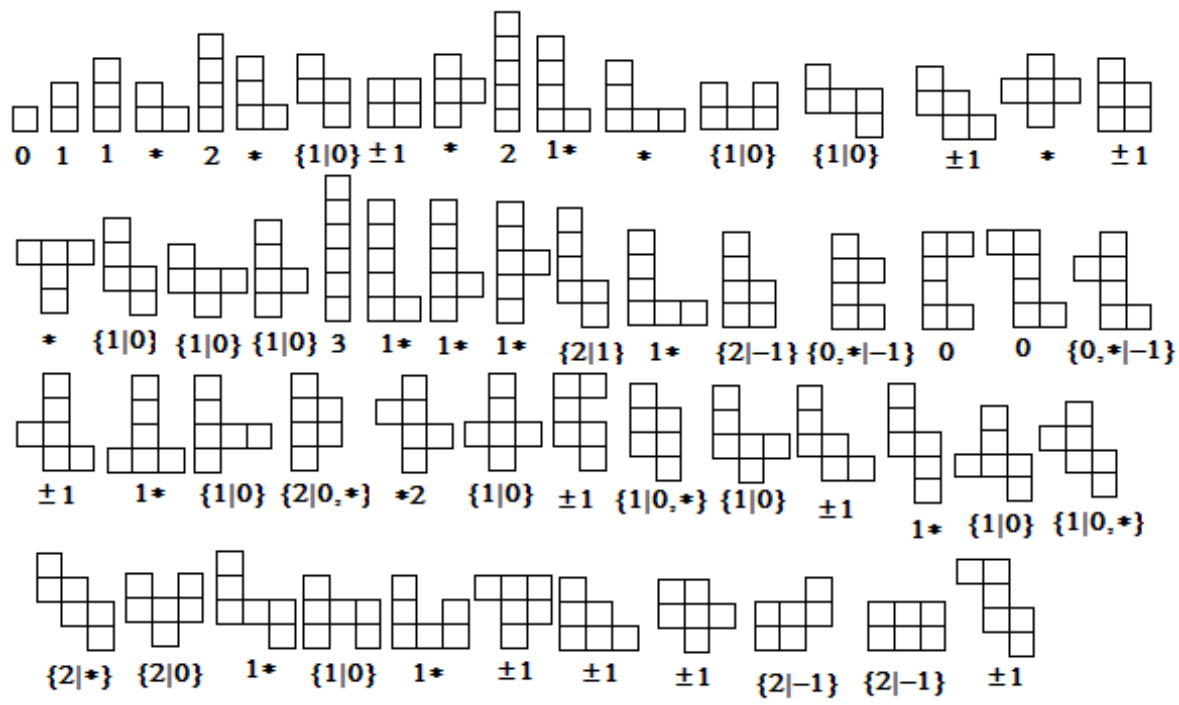

FiguRE 8. L-Tromping values for boards up to 6 squares.

TABLE 3. Values of L-Tromping for small rectangular boards.

\begin{tabular}{|c|c|c|c|c|c|}
\hline & 1 & 2 & 3 & 4 & 5 \\
\hline 1 & 0 & -1 & -1 & -2 & -2 \\
2 & 1 & \pm 1 & $\{2 \mid-1\}$ & $\left\{1^{*},\{2 \mid 0\} \mid-1^{*}\right\}$ & $\{0,\{3 \mid 0\} \mid 0,\{0 \mid-2\}\}$ \\
3 & 1 & $\{1 \mid-2\}$ & \pm 2 & $\mathrm{H}$ & $\mathrm{H}$ \\
4 & 2 & $\left\{1^{*} \mid\{0 \mid-2\},-1^{*}\right\}$ & $\mathrm{V}$ & $\mathrm{F}$ & $\mathrm{F}$ \\
5 & 2 & $\{0,\{2 \mid 0\} \mid 0,\{0 \mid-3\}\}$ & $\mathrm{V}$ & $\mathrm{F}$ & $\mathrm{F}$ \\
\hline
\end{tabular}

As can be seen in the dictionaries of Figures 8 and 9 , the value ${ }^{*} 2$ is attained several times on small boards of L-Tromping. The first time is on a board of only 6 squares. For domineering, on the other hand, it is not so easy to construct a board with value ${ }^{*} 2$. Such a board was recently constructed by G.C. Drummond-Cole [Dru]. It appears that the values of L-Tromping are hotter than those of our preceding games. If this is indeed the case in general, it may be due to the L-tromino, which can be used by both players. Thus the game resembles more an impartial game in which every nonzero value is hot.

L-Tromping also satisfies Proposition 2.6 of D-Tromping.

Here is another property of L-Tromping: 


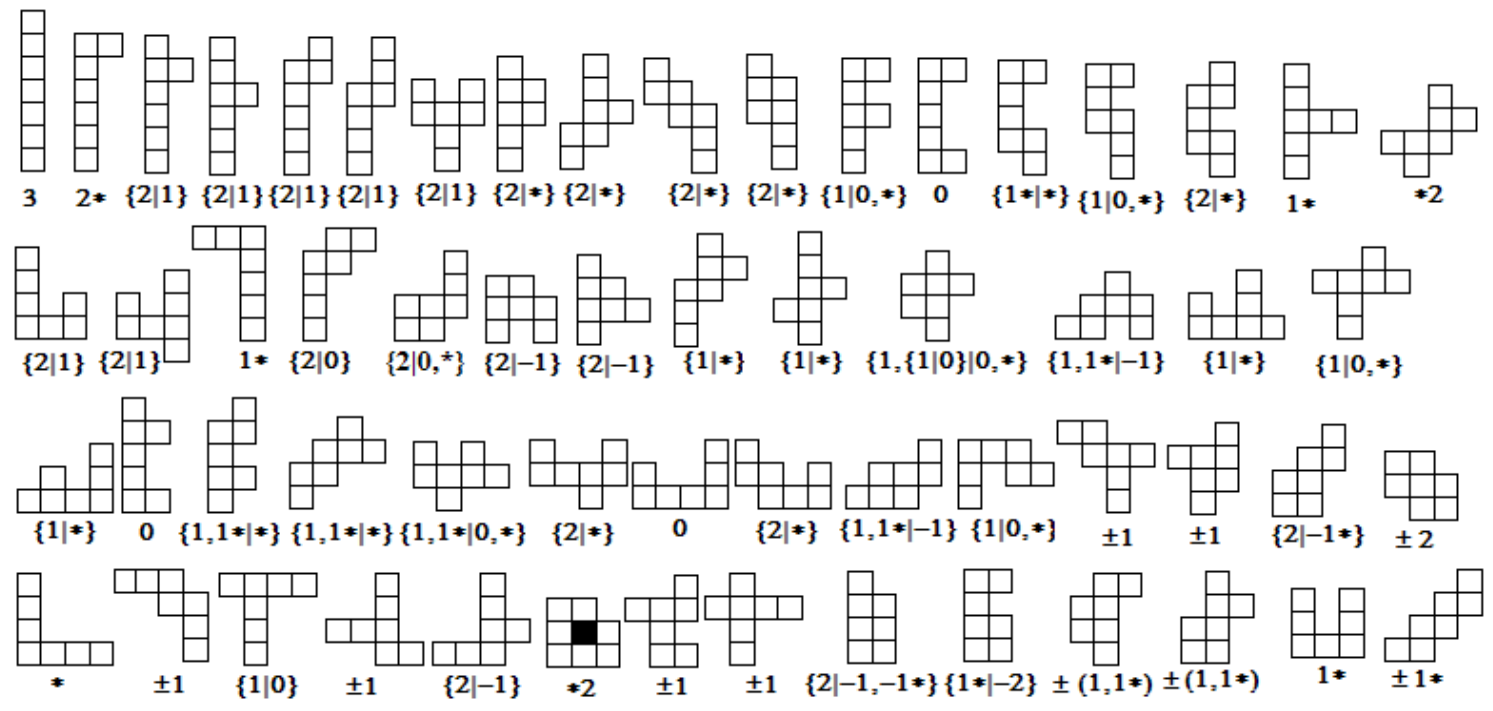

Figure 9. L-Tromping values for some boards with 7 squares.

Lemma 2.7. Let $B$ be a board and let $B^{\prime}$ be a subboard obtained from $B$ by removing either a single $k \times 1$ subboard $S$ of $B$, where $k \in\{2,3\}$ or an L-tromino. Then in L-Tromping, $B<0$ implies $B^{\prime} \ngtr 0$.

Proof. Suppose that Vertical begins tiling on $B$ with a $2 \times 1$ or $3 \times 1$ tile or with an L-tromino, so that $B^{\prime}$ results. If $B^{\prime}>0$, then Vertical can win as player I on $B$, so $B \| 0$ or $B>0$, a contradiction. Hence $B^{\prime} \ngtr 0$.

Remark 2.8. An analogous statement holds for Domineering, I-tromping, D-Tromping, by removing a $2 \times 1,3 \times 1, k \times 1(k \in\{2,3\})$ subboard, respectively.

\section{Connection with the Frobenius problem}

Definition 3.1. Let $n \in \mathbb{Z}_{>0}$, and $A \subset \mathbb{Z}_{>0}$ a finite set. We say that $A$ is a nonnegative integer basis for $\mathbb{Z}_{>n}$ if every integer greater than $n$ can be written as a linear nonnegative integer combination of elements of $A$.

Example. $A=\{4,5,6\}$. One can easily see that $A$ is a nonnegative basis for $\mathbb{Z}_{>7}$. Indeed, any nonnegative integer combination of elements of $A$ has the form $4 k_{1}+5 k_{2}+6 k_{3}$ for $k_{1}, k_{2}, k_{3} \in \mathbb{Z}_{\geq 0}$, not all of them zero: Considering the positive integers modulo 4 we get

- For $n$ of the form $4 k, k>0$, we use $k_{2}=k_{3}=0$.

- For $n$ of the form $4 k+1, k>0$, we use $k_{1}=k-1, k_{2}=1, k_{3}=0$.

- For $n$ of the form $4 k+2, k>0$, we use $k_{1}=k-1, k_{2}=0, k_{3}=1$.

- For $n$ of the form $4 k+3, k>1$, we use $k_{1}=k-2, k_{2}=1, k_{3}=1$.

It is clear that there can be more than one nonnegative basis for $\mathbb{Z}_{>n}, n \in \mathbb{Z}_{>0}$. For example, consider $\mathcal{B}_{1}=\{12, \ldots, 23\}$ and $\mathcal{B}_{2}=\{4,9,11,14\}$. The set $\mathcal{B}_{1}$ is a nonnegative integer basis for $\mathbb{Z}_{>11}$ by Proposition 3.2 below. Furthermore we can see that $\mathcal{B}_{2}$ is also a nonnegative integer basis for $\mathbb{Z}_{>11}$ (we can follow the preceding example, noting that $\mathcal{B}_{2}$ is a complete system of remainders modulo 4 ).

The following is a result on nonnegative bases. 
Proposition 3.2. If $k \in \mathbb{Z}_{>0}$, then the set $A=\{k, k+1, \ldots, 2 k-1\}$ forms a nonnegative basis for $\mathbb{Z}_{>k-1}$.

Proof. For any $n \in \mathbb{Z}_{\geq k}, n$ can be written uniquely in the form $n=q k+r$, with $q \in \mathbb{Z}_{\geq 0}$ and $k \leq r<2 k$, so $r \in A$.

For example, the set $\{3,4,5\}$ forms a nonnegative basis for $\mathbb{Z}_{>2}$.

While playing a game, a board $B$ will normally be divided into smaller boards. For example, in I-Tromping, Vertical can divide a $3 \times n$ board into two as soon as he performs his first move. On the other hand, Horizontal also has the power of dividing a board, by avoiding certain moves. For example, if the game takes place on a $3 \times 8$ board, Horizontal can divide such a board into two $3 \times 4$ subboards by not tiling squares from the fourth and fifth column in the same move.

The above observation shows that, when playing I-Tromping or D-Tromping, if Horizontal can divide an $m \times n$ board into subboards in which he wins, the whole board will be won by Horizontal.

More concretely, fix $m \in \mathbb{Z}_{>0}$ and let $A=\left\{n_{1}, n_{2}, \ldots, n_{k}\right\} \subset \mathbb{Z}_{>0}$ so that Horizontal wins I-Tromping or D-Tromping on the $m \times n_{i}$ board, $i=1, \ldots, k$. Then Horizontal wins on the $m \times n$ board, for $n=\sum_{i=1}^{k} a_{i} n_{i}$ and $a_{i} \in \mathbb{Z}_{\geq 0}$ for $i=1, \ldots, k$.

As an interesting connection with Number Theory, the largest integer $N$ for which there is no solution to the Diophantine equation $N=\sum_{i=1}^{k} x_{i} n_{i}$ in nonnegative integers $x_{i}$ is known as the Frobenius number for $A$. See [Guy], C7. Finding the Frobenius number for a given set of positive integers is known as the Frobenius problem (sometimes referred to as Frobenius coin problem or coin problem).

So in particular, if we can find a nonnegative integer basis $\mathcal{B}$ for $\mathbb{Z}_{\geq k}$ with each element $n_{i} \in \mathcal{B}$ representing a subboard $m \times n_{i}$ (for fixed $m$ ) that can be won by Horizontal, then Horizontal will win all $m \times n$ boards, where $n \geq k$. This is the use of nonnegative bases in our study. $[\mathrm{LMR}]$.

The above application relies on the following theorem, which is a generalization of a result implicit in

Theorem 3.3. Let $\mathcal{F}=\left\{F_{1}, \ldots, F_{k}\right\}$ be a set of boards such that $F_{i}$ is aligned to $F_{j}$ for all $i, j \in$ $\{1, \ldots, k\}$, and $F_{i}<0$ for all $i$. If $G$ is constructed by concatenating any finite number of copies of boards from $\mathcal{F}$, not necessarily distinct copies, then, for I-Tromping and D-Tromping, $G<0$.

Proof. The idea is to notice that Horizontal has the power to divide $G$ into smaller boards by refraining to tile more than one subboard. The following is a winning strategy for Horizontal: Divide $G$ into subboards and play exclusively on a given copy of a game, without ever tiling across the border of 2 adjacent subboards. Vertical is unable to play on more than one copy by alignment. In this way, Horizontal establishes vertical boundaries, which cannot be crossed by Vertical. Since $F_{i}<0$, for $i=1, \ldots, k$, Horizontal can force a win on each subboard. In this fashion, $G$ becomes the sum of the copies of elements of $\mathcal{F}$, and so Horizontal can win $G$.

Note that this method cannot be applied directly to L-Tromping, as each player is allowed to use an L-tromino, and so Horizontal no longer has the advantage of restricting the play to a given copy. Perhaps Theorem 3.3 still holds anyway.

\section{WHO WINS ON HORIZONTAL STRIPS?}

4.1. Results for particular boards. We will focus on games of the form $m \times n$, where $m=2,3,4,5$. The tables were computed using the plug-in for the CGS software. 
As mentioned earlier, we denote in the tables a win for Horizontal by $\mathrm{H}$, a win for Vertical by $\mathrm{V}$, and a win for the first player by $\mathrm{F}$.

We apply the following method: For fixed $m$, we try to find several values $n_{i}$ for which the games played on the $m \times n_{i}$ board are negative. Then by Theorem 3.3, concatenating any number of those boards will produce a negative game. If the $n_{i}$ s also form a nonnegative basis for $\mathbb{Z}_{>k}$ for some $k \in \mathbb{Z}_{>0}$, then we can determine the winner for a game played on an $m \times n$ board, $n>k$.

Note that Horizontal wins I-Tromping on $2 \times n$ boards for all $n \geq 3$, as Vertical cannot move at all on any such board. For $n \in\{1,2\}$, the first player loses. Of course all results for boards of the form $m \times n$ have a similar version for boards of the form $n \times m$, with the respective changes. More specifically, the board of the form $n \times m$ is the negative of the $m \times n$ board. If a board is a win for Horizontal, its negative is a win for vertical, and vice-versa. If a board is a win for the first (second) player, its negative is also a win for the first (second) player, respectively.

4.2. I-Tromping for $3 \times n$ boards. Many values found so far are fuzzy, as seen in Table 4 . More information is needed to determine the winner for an arbitrary $n$. However, since Horizontal wins in the $3 \times 6$ game, Theorem 3.3 implies that Horizontal wins for all boards of the form $3 \times 6 k$ for $k \in \mathbb{Z}_{\geq 1}$.

Remark 4.1. If we concatenate (in an aligned manner) a board $A$ that is won by Horizontal and a board $B$ that is won by the first player we obtain a board that is won by either Horizontal or the first player. This observation is made in [LMR], Table (2-3). Indeed if Horizontal plays first, he can start in $B$ and counter Vertical's moves in $A$. For instance, since $19=6+13$, we can conclude the winner of I-Tromping on the $3 \times 19$ board is either Horizontal or the first player, for Horizontal wins the $3 \times 6$ board and the first player wins the $3 \times 13$ board. Similarly, from Table 4 we conclude that either the first player or Vertical wins I-Tromping on the $3 \times m$ board, where

$m \in\left\{3+6 j, 4+6 j, 5+6 j, 7+6 k, 9+6 k, 10+6 k, 11+6 k, 13+6 l, 14+6 l, 15+6 l, 16+6 l, 17+6 l \mid j, k, l \in \mathbb{Z}_{\geq 3}, k, l \in \mathbb{Z}_{\geq 2}\right\}$

Remark 4.2. Notice that we cannot tell who wins in the concatenation of a board that is won by Vertical and one that is won by the first player since Horizontal can cross the vertical boundaries of these two boards.

TABLE 4. I-Tromping values for $3 \times n$ boards

\begin{tabular}{|c|c|c|c|c|c|c|c|c|c|c|c|c|c|c|c|c|c|c|}
\hline$n$ & 1 & 2 & 3 & 4 & 5 & 6 & 7 & 8 & 9 & 10 & 11 & 12 & 13 & 14 & 15 & 16 & 17 & 18 \\
\hline & $\mathrm{V}$ & $\mathrm{V}$ & $\mathrm{F}$ & $\mathrm{F}$ & $\mathrm{F}$ & $\mathrm{H}$ & $\mathrm{F}$ & $\mathrm{V}$ & $\mathrm{F}$ & $\mathrm{F}$ & $\mathrm{F}$ & $\mathrm{H}$ & $\mathrm{F}$ & $\mathrm{F}$ & $\mathrm{F}$ & $\mathrm{F}$ & $\mathrm{F}$ & $\mathrm{H}$ \\
\hline
\end{tabular}

4.3. I-Tromping for $4 \times n$ boards. We find that the boards for $n \in\{6, \ldots, 11\}$ have negative values. Further, $\{6, \ldots, 11\}$ forms a nonnegative basis for $\mathbb{Z}_{>5}$ by Proposition 3.2. Hence, Horizontal wins I-Tromping on $4 \times n$ boards for all $n \geq 6$, by Theorem 3.3. Table 5 shows the winner for $n<6$.

TABLE 5. I-Tromping values for $4 \times n$ boards

\begin{tabular}{|c|c|c|c|c|c|}
\hline$n$ & 1 & 2 & 3 & 4 & 5 \\
\hline & $\mathrm{V}$ & $\mathrm{V}$ & $\mathrm{F}$ & $\mathrm{F}$ & $\mathrm{F}$ \\
\hline
\end{tabular}

4.4. I-Tromping for $5 \times n$ boards. By Table $6, H$ can win for all $n \in\{6, \ldots, 11\}$, so Proposition 3.2 implies that Horizontal can win all games with $n \geq 6$. The table also shows who wins for $n<6$.

TABLE 6. I-Tromping values for $5 \times n$ boards

\begin{tabular}{|c|c|c|c|c|c|c|c|c|c|c|c|}
\hline$n$ & 1 & 2 & 3 & 4 & 5 & 6 & 7 & 8 & 9 & 10 & 11 \\
\hline & $\mathrm{V}$ & $\mathrm{V}$ & $\mathrm{F}$ & $\mathrm{F}$ & $\mathrm{F}$ & $\mathrm{H}$ & $\mathrm{H}$ & $\mathrm{H}$ & $\mathrm{H}$ & $\mathrm{H}$ & $\mathrm{H}$ \\
\hline
\end{tabular}


4.5. D-Tromping for $2 \times n$ boards. By computation, $2 \times n$ boards have negative values for $n \in$ $\{4,9,11,14\}$. This set forms a nonnegative basis for $\mathbb{Z}_{>11}$. Table 7 shows the values for boards of width less than 12. Therefore, we know who wins D-Tromping on boards of the form $2 \times n$ for all $n \geq 1$.

TABLE 7. D-Tromping values for $2 \times n$ boards

\begin{tabular}{|c|c|c|c|c|c|c|c|c|c|c|c|}
\hline$n$ & 1 & 2 & 3 & 4 & 5 & 6 & 7 & 8 & 9 & 10 & 11 \\
\hline & $\mathrm{V}$ & $\mathrm{F}$ & $\mathrm{F}$ & $\mathrm{H}$ & $\mathrm{F}$ & $\mathrm{F}$ & $\mathrm{F}$ & $\mathrm{H}$ & $\mathrm{H}$ & $\mathrm{F}$ & $\mathrm{H}$ \\
\hline
\end{tabular}

4.6. D-Tromping for $3 \times n$ boards. The game has negative values for $n \in\{4,5,6\}$. Hence by the example in Section 3 Horizontal also wins the game for $n>7$. Table 8 gives the values for $n \leq 7$. So the winner of D-Tromping on boards of the form $3 \times n$ is known for all $n \geq 1$.

TABLE 8. D-Tromping values for $3 \times n$ boards

\begin{tabular}{|c|c|c|c|c|c|c|c|}
\hline$n$ & 1 & 2 & 3 & 4 & 5 & 6 & 7 \\
\hline & $\mathrm{V}$ & $\mathrm{F}$ & $\mathrm{F}$ & $\mathrm{H}$ & $\mathrm{H}$ & $\mathrm{H}$ & $\mathrm{F}$ \\
\hline
\end{tabular}

4.7. D-Tromping for $4 \times n$ boards. Table 9 summarizes the results we have obtained so far. Since Horizontal wins the $4 \times 6$ board, Theorem 3.3 implies that Horizontal also wins on all boards of the form $4 \times 6 k, k \in \mathbb{Z}_{>0}$. Furthermore, Remark 4.1 in Section 4.2 implies that either Horizontal or the first player is the winner of D-Tromping for boards of the form $4 \times m$, where $m \in\left\{4+6 k, 5+6 k, 7+6 k \mid k \in \mathbb{Z}_{\geq 1}\right\}$.

TABLE 9. D-Tromping values for $4 \times n$ boards

\begin{tabular}{|c|c|c|c|c|c|c|c|}
\hline$n$ & 1 & 2 & 3 & 4 & 5 & 6 & 7 \\
\hline & $\mathrm{V}$ & $\mathrm{V}$ & $\mathrm{V}$ & $\mathrm{F}$ & $\mathrm{F}$ & $\mathrm{H}$ & $\mathrm{F}$ \\
\hline
\end{tabular}

4.8. L-Tromping for $2 \times n$ boards. Table 10 shows who can win for $1 \leq n \leq 15$.

TABLE 10. L-Tromping values for $2 \times n$ boards

\begin{tabular}{|c|c|c|c|c|c|c|c|c|c|c|c|c|c|c|c|}
\hline$n$ & 1 & 2 & 3 & 4 & 5 & 6 & 7 & 8 & 9 & 10 & 11 & 12 & 13 & 14 & 15 \\
\hline & $\mathrm{V}$ & $\mathrm{F}$ & $\mathrm{F}$ & $\mathrm{F}$ & $\mathrm{F}$ & $\mathrm{F}$ & $\mathrm{F}$ & $\mathrm{F}$ & $\mathrm{F}$ & $\mathrm{F}$ & $\mathrm{F}$ & $\mathrm{F}$ & $\mathrm{F}$ & $\mathrm{F}$ & $\mathrm{H}$ \\
\hline
\end{tabular}

4.9. L-Tromping for $3 \times n$ boards. Table 11 summarizes the winner of L-Tromping for $3 \times n$ boards, $n \leq 12$.

TABLE 11. L-Tromping values for $3 \times n$ boards

\begin{tabular}{|c|c|c|c|c|c|c|c|c|c|c|c|c|}
\hline$n$ & 1 & 2 & 3 & 4 & 5 & 6 & 7 & 8 & 9 & 10 & 11 & 12 \\
\hline & $\mathrm{V}$ & $\mathrm{F}$ & $\mathrm{F}$ & $\mathrm{H}$ & $\mathrm{H}$ & $\mathrm{H}$ & $\mathrm{F}$ & $\mathrm{H}$ & $\mathrm{H}$ & $\mathrm{H}$ & $\mathrm{H}$ & $\mathrm{H}$ \\
\hline
\end{tabular}

Although we are not certain that Theorem 3.3 holds for L-Tromping, the theorem may be true for $3 \times n$ boards. The key here is the geometry of the board. Horizontal seems to have the upper hand by tiling squares in the second row, by which he reserves some squares in the first and third row for future moves. 
4.10. Results on M-Tromping. Motivated by L-Tromping, we create a game in which each of the two players is only allowed to use L-trominoes. Since the sets of moves for each player are identical, M-Tromping is an iMpartial game. The Sprague-Grundy theory tells us that its values are nimbers. So for each game of M-Tromping $G$, we have,

$$
G=\left\{* a_{1}, * a_{2}, \cdots, * a_{n} \mid * a_{1}, * a_{2}, \cdots, * a_{n}\right\}=\operatorname{mex}\left\{* a_{1}, * a_{2}, \cdots, * a_{n}\right\},
$$

where for any set $S \subsetneq \mathbb{Z}_{\geq 0}$, mex $S=\min \mathbb{Z}_{\geq 0} \backslash S=$ smallest nonnegative integer not in $S$.

We present the value of M-Tromping for small rectangular boards in Table 12. Note that such a table is symmetric, since every nimber is its own negative.

TABLE 12. Sprague-Grundy values for M-Tromping played on the $m \times n$ board.

\begin{tabular}{|c||c|c|c|c|c|c|}
\hline$m \backslash n$ & 1 & 2 & 3 & 4 & 5 & 6 \\
\hline \hline 1 & 0 & 0 & 0 & 0 & 0 & 0 \\
2 & 0 & 1 & 2 & 0 & 3 & 1 \\
3 & 0 & 2 & 0 & 1 & 2 & 2 \\
4 & 0 & 0 & 1 & 0 & 1 & 0 \\
5 & 0 & 3 & 2 & 1 & 0 & 1 \\
6 & 0 & 1 & 2 & 0 & 1 & 1 \\
\hline
\end{tabular}

Let $m \in \mathbb{Z}_{>0}$ and $n \in \mathbb{Z}_{\geq 0}$. Denote by $G(m, n)$ the game of M-Tromping played on an $m \times n$ rectangular board. Furthermore, let $G_{1}(m, n)$ be the game of M-Tromping played on an $m \times n$ board with two opposite corners removed (say, the upper-left and lower-right corner) and let $G_{2}(m, n)$ be the game of M-Tromping played on an $m \times n$ board with the first and last square of the first row removed. It is convenient to define $G(m, 0)=G_{1}(m, 0)=G_{1}(m, 1)=G_{2}(m, 0)=G_{2}(m, 1)=0$. We prove,

Theorem 4.3. The games $G(2, n), G_{1}(2, n+1)$, and $G_{2}(2, n+1)$ have the same value for all $n \in \mathbb{Z}_{\geq 0}$.

Before proving Theorem 4.3, we need some notation to describe moves on $G(2, n), G_{1}(2, n+1)$, and $G_{2}(2, n+1)$. Label the squares of both rows of $G(2, n)$ and $G_{1}(2, n+1)$ from left to right with numbers from $[n]=\{1,2, \ldots, n\}$ in increasing order. Similarly, label the squares in the first row of $G_{2}(2, n+1)$ with $\{2,3,4, \ldots, n\}$ in increasing order from left to right and the squares in the second row with $[n+1]$ increasingly from left to right (see Figures 10 and 11.) In any of these boards let $(a, a+1 ; a+1)$ represent the move where the L-Triomino is covering the squares labeled $a, a+1$ from the first row and $a+1$ from the second one, with $a, a+1 \in[n]$. Define $(a, a+1 ; a),(a ; a, a+1),(a+1 ; a, a+1)$ similarly, where the semicolon is used to separate the labels from different rows.

Proof. It is enough to show that $G(2, n)+G_{1}(2, n+1)=0$ and $G(2, n)+G_{2}(2, n+1)=0$, for $n>0$.

We proceed by induction on $n$. For $n=0,1$, all three games have value 0 and for $n=2$, all have value $*$. For $n>2$, suppose that for $m<n, G(2, m)+G_{1}(2, m+1)=0$ and $G(2, m)+G_{2}(2, m+1)=0$.

The rules below, depicted in Figure 10, guarantee a win for the second player in $G(2, n)+G_{1}(2, n+1)$ : If the first player's first move is

(1) $(k ; k, k+1)$ in $G(2, n)$ then move $(k ; k, k+1)$ in $G_{1}(2, n+1)$, for $1 \leq k<n$.

(2) $(k, k+1 ; k)$ in $G(2, n)$ then move $(k ; k, k+1)$ in $G_{1}(2, n+1)$, for $1 \leq k<n$.

(3) $(k-1, k ; k)$ in $G(2, n)$ then move $(k-1, k ; k)$ in $G_{1}(2, n+1)$, for $1<k \leq n$.

(4) $(k ; k-1, k)$ in $G(2, n)$ then move $(k-1, k ; k)$ in $G_{1}(2, n+1)$, for $1<k \leq n$.

(5) $(k ; k, k+1)$ in $G_{1}(2, n+1)$ then move $(k ; k, k+1)$ in $G(2, n)$, for $1 \leq k<n$.

(6) $(k-1, k ; k)$ in $G_{1}(2, n+1)$ then move $(k-1, k ; k)$ in $G(2, n)$, for $1<k \leq n$.

Now each player has moved exactly once, and the two boards have been divided into at most four subboards: two rectangular boards $T_{1}$ and $T_{2}$ with a corner square removed, one $G(2, m)$, and one 
$G_{1}(2, m+1)$ for some $0 \leq m<n$ (these last two could be empty). By the induction hypothesis, $G(2, m)+G_{1}(2, m+1)=0$. Since $T_{1}$ and $T_{2}$ have the same shape (after rotating and flipping if necessary), $T_{1}+T_{2}=0$. So after the first move, the second player can turn the game into a zero game.

The rules for the remaining possible first moves of the first player in $G(2, n)+G_{1}(2, n+1)$ now follow:

(7) $(k-1, k ; k+1)$ in $G_{1}(2, n+1)$ then move $(k, k+1 ; k)$ in $G(2, n)$, for $1<k<n$.

(8) $(k-1 ; k, k+1)$ in $G_{1}(2, n+1)$ then move $(k ; k-1, k)$ in $G(2, n)$, for $1<k<n$.

Now each player has moved exactly once, and the two boards have been divided into at most four subboards: two rectangular boards $T_{1}$ and $T_{2}$ with a corner square removed, one $G(2, m)$, and one $G_{2}(2, m+1)$ for some $0 \leq m<n$ (these last two could be empty). By the induction hypothesis, $G(2, m)+G_{2}(2, m+1)=0$. As above, $T_{1}$ and $T_{2}$ have the same shape, hence $T_{1}+T_{2}=0$. So after the first move, the second player can turn the game into a zero game.

Thus $G(2, n)+G_{1}(2, n+1)=0$.

(1)

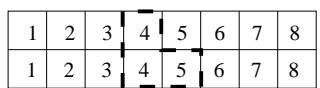

(2)

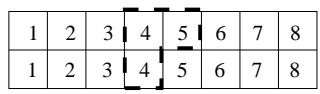

(3)

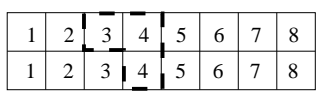

(4)

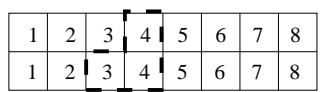

(5)

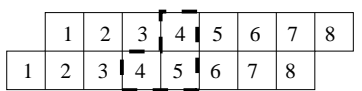

(6)

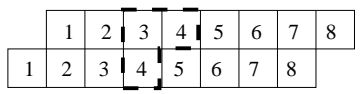

(7)

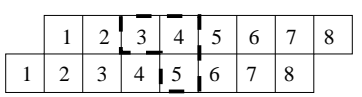

(8)

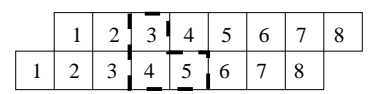

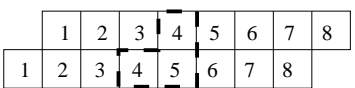
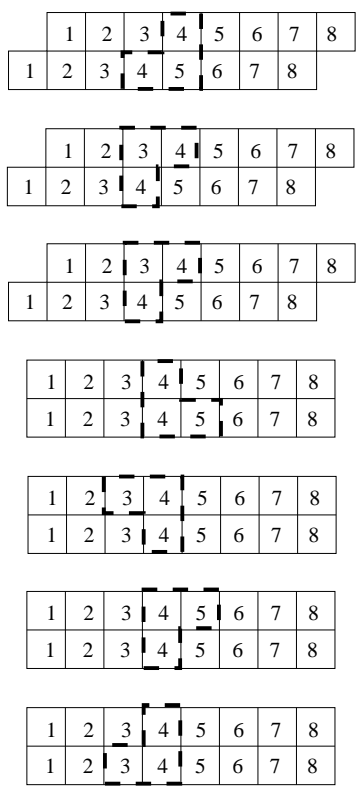

FiguRE 10. Second player's response in the game $G(2,8)+G_{1}(2,9)$ according to the rules (1)-(8) with $k=4$. The first player has 8 possible first moves, one for each pair of the 4 orientations of the L-tromino and the 2 boards $G, G_{1}$. First player's moves are depicted in the first column of boards; second player's response in the second column.

The rules below, depicted in Figure 11, guarantee a win for the second player in $G(2, n)+G_{2}(2, n+1)$ : If the first player's first move is

(1') $(k ; k, k+1)$ in $G(2, n)$ then move $(k+1 ; k, k+1)$ in $G_{2}(2, n+1)$, for $1 \leq k<n$.

$\left(2^{\prime}\right)(k, k+1 ; k)$ in $G(2, n)$ then move $(k+1 ; k, k+1)$ in $G_{2}(2, n+1)$, for $1 \leq k<n$.

$\left(3^{\prime}\right)(k-1, k ; k)$ in $G(2, n)$ then move $(k ; k, k+1)$ in $G_{2}(2, n+1)$, for $1<k \leq n$.

(4') $(k ; k-1, k)$ in $G(2, n)$ then move $(k ; k, k+1)$ in $G_{2}(2, n+1)$, for $1<k \leq n$.

(5') $(k+1 ; k, k+1)$ in $G_{2}(2, n+1)$ then move $(k ; k, k+1)$ in $G(2, n)$, for $1 \leq k<n$.

$\left(6^{\prime}\right)(k ; k, k+1)$ in $G_{2}(2, n+1)$ then move $(k ; k-1, k)$ in $G(2, n)$, for $1<k \leq n$. 
(1')

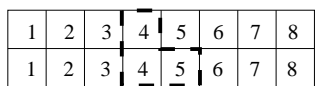

(2')

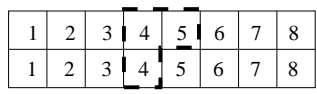

(3')

\begin{tabular}{|l|l|l|r|l|l|l|l|}
\hline 1 & 2 & $\overline{3}$ & $\overline{4}$ & 5 & 6 & 7 & 8 \\
\hline 1 & 2 & 3 & 4 & 5 & 6 & 7 & 8 \\
\hline
\end{tabular}

(4')

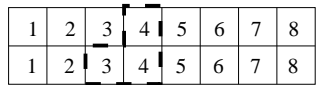

$\left(5^{\prime}\right)$

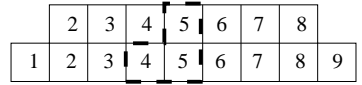

(6')

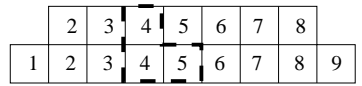

(7')

\begin{tabular}{|l|l|l|l|l|l|l|l|l|}
\cline { 2 - 8 } \multicolumn{1}{l|}{} & 2 & 3 & 4 & 5 & 6 & 7 & 8 & \\
\hline 1 & 2 & 3 & 4 & 5 & 6 & 7 & 8 & 9 \\
\hline
\end{tabular}

(8')

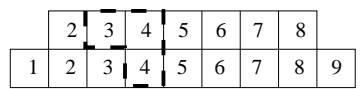

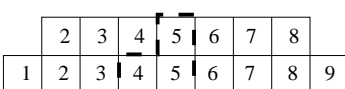
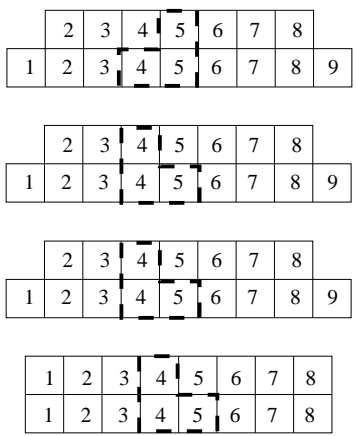

\begin{tabular}{|l|l|l|l|l|l|l|l|}
\hline 1 & 2 & 3 & 4 & 5 & 6 & 7 & 8 \\
\hline 1 & 2 & 3 & 4 & 5 & 6 & 7 & 8 \\
\hline
\end{tabular}

\begin{tabular}{|l|l|l|l|l|l|l|l|}
\hline 1 & 2 & 3 & 4 & 5 & 6 & 7 & 8 \\
\hline 1 & 2 & 3 & 4 & 5 & 6 & 7 & 8 \\
\hline
\end{tabular}

\begin{tabular}{|l|l|l|l|l|l|l|l|}
\hline 1 & 2 & 3 & 4 & 5 & 6 & 7 & 8 \\
\hline 1 & 2 & 3 & 4 & 5 & 6 & 7 & 8 \\
\hline
\end{tabular}

Figure 11. Second player's response in the game $G(2,8)+G_{2}(2,9)$ according to the rules $\left(1^{\prime}\right)-\left(8^{\prime}\right)$ with $k=4$. The first player has 8 possible first moves, one for each pair of the 4 orientations of the L-tromino and the 2 boards $G, G_{2}$. First player's moves are depicted in the first column of boards; second player's response in the second column.

Now, each player has moved exactly once, and the two boards have been divided into at most 4 subboards: two rectangular boards $T_{1}$ and $T_{2}$ with a square removed, one $G(2, m)$, and one $G_{1}(2, m+1)$ for some $0 \leq m<n$ (these last two could be empty). By the inductive hypothesis, $G(2, m)+G_{1}(2, m+$ 1 ) $=0$ and since $T_{1}$ and $T_{2}$ have the same shape (after rotating and flipping if necessary), then $T_{1}+T_{2}=0$. So after the first move, the second player can guarantee that the game becomes a zero game.

The rules for the remaining possible first moves of the first player in $G(2, n)+G_{2}(2, n+1)$ now follow

$\left(7^{\prime}\right)(k, k+1 ; k)$ in $G_{2}(2, n+1)$ then move $(k-1, k ; k)$ in $G(2, n)$, for $1<k \leq n$.

$\left(8^{\prime}\right)(k-1, k ; k)$ in $G_{2}(2, n+1)$ then move $(k-1, k ; k-1)$ in $G(2, n)$, for $2<k \leq n$.

Each player has now moved exactly once, and the two boards have been divided into at most four subboards: two rectangular boards $T_{1}$ and $T_{2}$ with a square removed, one $G(2, m)$, and one $G_{2}(2, m+1)$ for some $0 \leq m<n$ (these last two could be empty). By the inductive hypothesis, $G(2, m)+G_{2}(2, m+$ $1)=0$ and since $T_{1}$ and $T_{2}$ have the same shape (after rotating and flipping if necessary), then $T_{1}+T_{2}=0$. So after the first move, the second player can guarantee that the game becomes a zero game.

Thus $G(2, n)+G_{2}(2, n+1)=0$. This completes the proof.

As noted above, M-Tromping is an impartial game and therefore the winner of this game is either the first or second player. For example, $G(2,3)$ is a first-player win, and $G(2,4)$ is a second-player win. Curiously, however, by just removing a corner from a $2 \times n$ board, M-Tromping becomes a first-player game for all $n>1$. We prove this in the following theorem.

Theorem 4.4. For $n>1$, let $B(n)$ be the value of $M$-Tromping played on a $2 \times n$ board with one corner removed. Then $B(n) \| 0$ for all $n$. 
Proof. Without loss of generality, we assume that the square removed is the lower-right corner. After labeling both columns of the board with numbers from $[n]$ in increasing order, we note that the statement is trivial for $n=2$. For $n>2$, we recognize two cases,

$n$ is odd: The first player moves $\left(\frac{n+1}{2} ; \frac{n+1}{2}-1, \frac{n+1}{2}\right)$, this will bisect the board into two pieces with value $B\left(\frac{n+1}{2}-1\right)$. After this move, the value of the game is $B\left(\frac{n+1}{2}-1\right)+B\left(\frac{n+1}{2}-1\right)$, which is a zero-game, as $B\left(\frac{n+1}{2}-1\right)$ is its one negative. Therefore the first player (who is the second to play on $\left.B\left(\frac{n+1}{2}-1\right)+B\left(\frac{n+1}{2}-1\right)\right)$ can win the game.

$n$ is even: The first player moves $\left(\frac{n}{2}, \frac{n}{2}+1 ; \frac{n}{2}\right)$. This will split the original board into two pieces: $G\left(2, \frac{n}{2}-1\right)$ and $G_{1}\left(2, \frac{n}{2}\right)$, respectively. By Theorem 4.3, these two pieces have the same value when playing M-Tromping, and so its sum is 0 . Hence the first player (who is the second to play on $\left.G\left(2, \frac{n}{2}-1\right)+G_{1}\left(2, \frac{n}{2}\right)\right)$ can win the game.

4.11. Some families of values. When playing I-Tromping or D-Tromping on square boards, notice that the sets of moves for Horizontal and Vertical are negatives of one another. So these games are either a first or second-player win. Notice that the winner of both I-Tromping and D-Tromping on the $5 \times 5$ board is the first player. In contrast, it is shown in [Con] page 116 that Domineering on a $5 \times 5$ board is a second player win. We also note that Tables 1 and 2 are antisymmetric, since the value of a game on a board $B$ is the negative of the game on board $B^{\prime}$, where $B^{\prime}$ is the rotation of $B$ by $90^{\circ}$ about its center (clockwise or counterclockwise).

Finally, in addition to the dictionaries of values already provided, we present in Figure 12 some patterns of boards that have a clearly discernible pattern of values. One can prove the validity of some of these patterns by using the properties that were pointed out in Propositions 2.4 and 2.6. For example, to prove by induction the validity of the first sequence of I-Tromping, we use Proposition 2.4 (a) and (b), and the well-known fact that $*+*+\cdots+*$ is 0 if we add an even number of stars, and $*$ if we add an odd number of them.

The last two lines of Figure 12 constitute a sequence of values for L-Tromping. The values obtained are,

$$
*, \downarrow, \underbrace{\{\downarrow \mid-1\},-1,-1^{*},-1 \downarrow}, \underbrace{\{-1 \downarrow \mid-2\},-2,-2^{*},-2 \downarrow}, \underbrace{\{-2 \downarrow \mid-3\},-3,-3^{*},-3 \downarrow} \cdots
$$

The emerging pattern is a four-term block of the form $\{(1-a) \downarrow \mid-a\},-a,-a^{*},-a \downarrow$ for $a \in \mathbb{Z}_{\geq 1}$ increasing in steps of 1 .

We remark, in passing, that the "double cross" in Fig 12 (second board from left in first row of LTromping) has value $\{\{0 \mid-1\}, * \mid *, 0,\{0 \mid-1\}\}=\{* \mid 0,\{0 \mid-1\}\}$ (by domination) $=\{* \mid 0\}$ (by reversibility) $=\downarrow$. Note that, perhaps a bit counterintuitively, the best opening move for Horizontal is to tile with a domino the middle 2 squares.

\section{Concluding Remarks}

- We proved some properties of I-Tromping (Proposition 2.4) which generalize those of Domineering (Proposition 2.6). This may lead one to think that there could be some isomorphism between I-Tromping and Domineering. We believe, however, that this is not the case. In particular, we propose that there is no position in I-Tromping with value

$$
\pm\left(0,\left\{\{2 \mid 0\}, 2+_{2} \mid\{2 \mid 0\},-2\right\}\right),
$$

which is the value of a $4 \times 4$ board in Domineering.

- It is natural to generalize the results of this paper to larger polyominoes. Results of the form of Proposition 2.4 grow stronger with increasing size of the smallest participating polyomino. 


\section{I-Tromping}

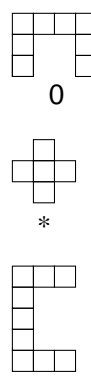

0
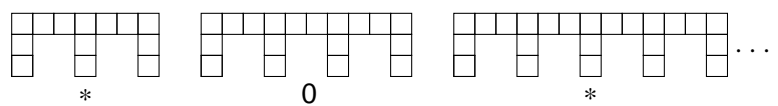

Period 2
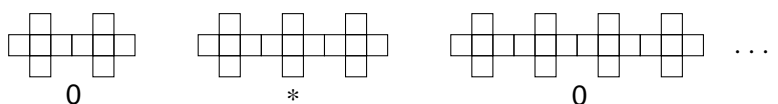

Period 2
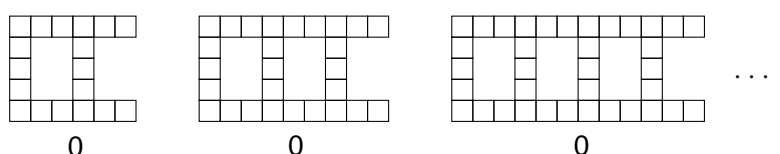

Period 1

\section{D-Tromping}

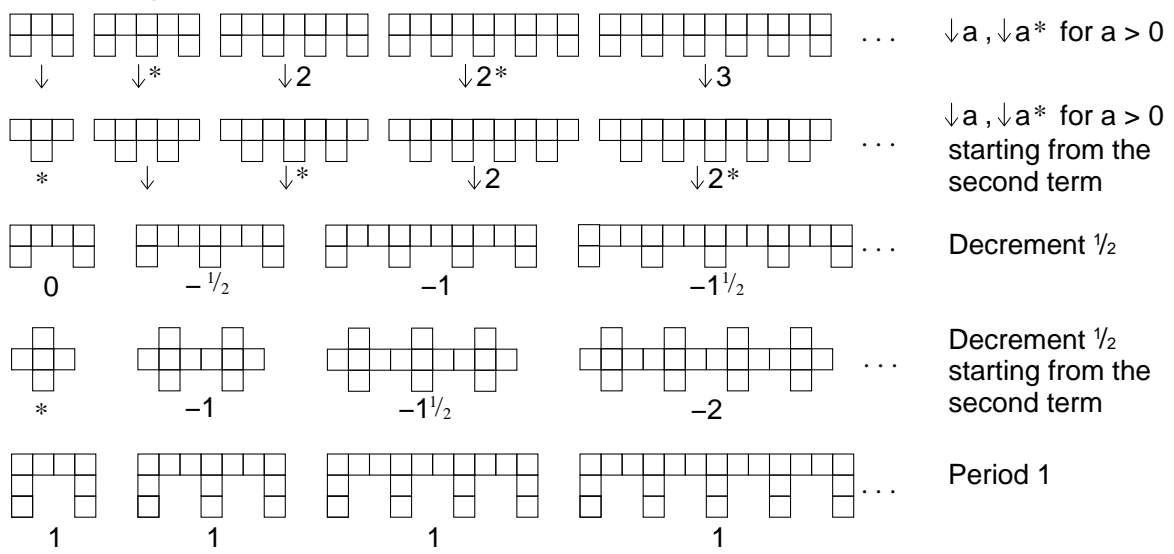

L-Tromping
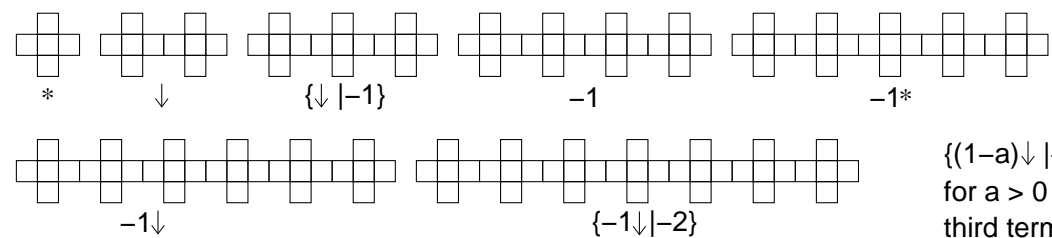

$\{(1-\mathrm{a}) \downarrow \mid-\mathrm{a}\},-\mathrm{a},-\mathrm{a}^{*},-\mathrm{a} \downarrow$

for $a>0$ starting from the

$\{-1 \downarrow \mid-2\}$

third term

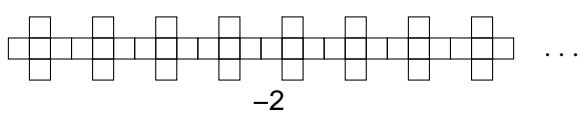

FIgURE 12. Families of patterns.

- The games presented here were analyzed for normal play; that is, the player making the last move wins. One can also analyze these games for misère play, where the player making the last move loses, but then the usefulness of sums is lost.

- For some of the boards, we need more experimental computations. But, since we are dealing with a very particular kind of board (horizontal strips), perhaps some heuristic techniques can be used.

- We do not know whether Theorem 3.3 can be applied in general for L-Tromping, although this seems plausible.

- M-Tromping on $2 \times m$ boards has interesting properties: while removing two corners basically does not change the game (see Theorem 4.3 for a precise statement), removing a single corner makes the game fuzzy. 


\section{REFERENCES}

[ANW] M. Albert, R. J. Nowakowski and D. Wolfe [2007], Lessons in Play: An Introduction to Combinatorial Game Theory, A K Peters.

[BCG] E. R. Berlekamp, J. H. Conway and R. K. Guy [2001-2004], Winning Ways for your Mathematical Plays, Vol. 1-4, A K Peters, Wellesley, MA, 2nd edition: vol. 1 (2001), vols. 2, 3 (2003), vol. 4 (2004).

[BUvdH] D.M. Breuker, J.W.H.M Uiterwijk, and H.J. van den Herik, "Solving $8 \times 8$ Domineering," Theoretical Computer Science 230 (2000) 195-206.

[Con] J.H. Conway, On Numbers and Games, Second Edition, AK Peters Ltd, 2001.

[Con77] _ "All Games Bright and Beautiful," The American Mathematical Monthly 84 (1977) 417-434.

[Dru] G.C. Drummond-Cole, "Positions of Value *2 in Generalized Domineering and Chess," INTEGERS, Electr. J. of Combinat. Number Theory 5, \#G6, 13pp., Comb. Games Sect. http://www.integers-ejcnt.org/vol5.html

[Gar] M. Gardner, "Mathematical Games" column, Scientific American 230(2) (February 1974) 106-108.

[Gar86] - "Cram, bynum and quadraphage," in Knotted Doughnuts and Other Mathematical Entertainments, Ch. 19, W.H. Freeman and Company, 1986.

[Guy] R.K. Guy, Unsolved Problems in Number Theory, 3rd edition, Springer, New York, 2004.

[LMR] M. Lachmann, C. Moore, and I. Rapaport, "Who Wins Domineering on Rectangular Boards?" in More Games of No Change, MSRI Publications, Vo. 42, 2002.

[Sie] A. Siegel, Combinatorial Game Suite, http://cgsuite.sourceforge.net/ (June 2004).

Department of Mathematics, Cornell University, IthacA, NY 14853

E-mail address: sabr@math.cornell.edu

Department of Computer Science and Applied Mathematics, Weizmann Institute Of Science, Rehovot, ISRAEL 76100

E-mail address: fraenkel@wisdom.weizmann.ac.il 